\title{
TMEM16F Regulates Baseline Phosphatidylserine Exposure and Cell Viability in Human Embryonic Kidney Cells
}

\author{
Laura K. Schenk Ulf Schulze Sebastian Henke Thomas Weide \\ Hermann Pavenstädt \\ Department of Nephrology, Hypertension and Rheumatology, University Hospital \\ Muenster, Muenster, Germany
}

\section{Key Words}

TMEM16F • Ano6 • Phosphatidylserine • Viability • FACS • AKT • ERK • HEK293

\begin{abstract}
Background / Aims: TMEM16F is a transmembrane protein from a conserved family of $\mathrm{Ca}^{2+}$-activated proteins, which is highly expressed in several tissues. TMEM16F confers phospholipid scramblase activity and $\mathrm{Ca}^{2+}$-activated electrolyte channel activity. Potentially thereby, TMEM16F is involved in cell cycle control and apoptotic signaling. The present study evaluated the role of TMEM16F on cell proliferation and viability in Human Embryonic Kidney cells. Methods: An inducible knockdown of TMEM16F was generated and markers of apoptosis and proliferation were assessed via flow cytometry, western blotting and MTT uptake assay under different conditions. Results: TMEM16F knockdown resulted in attenuated growth of HEK293 cells. This observation correlated with an increased phosphatidylserine exposure and a decreased fraction of viable cells. Interestingly, the cells were not sensitized to Staurosporine- induced cell death. Western blot analyses displayed a parallel activation of pro- and antiapoptotic signaling pathways: Caspase 3 cleavage and Cyclin D1 abundance were simultaneously increased. Furthermore, knockdown of TMEM16F led to activation of AKT signaling. Conclusion: TMEM16F modifies viability of Human Embryonic Kidney cells via its function as a phospholipid scramblase and activation of AKT signaling pathways.
\end{abstract}

\section{Introduction}

TMEM16F belongs to the conserved TMEM16 protein family. The ten family members, TMEM16A-K, also known as Anoctamins (Anoctamin 1-10), are transmembrane proteins with ten transmembrane domains and a conserved $\mathrm{Ca}^{2+}$-activated domain.

TMEM16F is expressed in many different types of human tissue, e.g. respiratory and gastrointestinal epithelia, osteoblasts, lymphocytes, platelets, vasculature and the kidney. In platelets and osteoblasts TMEM16F acts as a small-conductance $\mathrm{Ca}^{2+}$-activated nonselective 


\section{Cellular Physiology Cell Physiol Biochem 2016;38:2452-2463 \\ \begin{tabular}{l|l} 
DOI: 10.1159/000445596 & $\begin{array}{l}\text { O 2016 The Author(s). Published by S. Karger AG, Basel } \\
\text { www.karger.com/cpb }\end{array}$
\end{tabular} \\ Schenk et al.: TMEM16F HEK Viability}

cation channel permeating both mono- and divalent cations [1-3]. Similar TMEM16F functionality has been shown for HEK293 cells.

Electrophysiological properties of TMEM16F isoforms in different cells types have been characterized in detail [1-12]. Moreover, TMEM16F is involved in the regulation of phosphatidylserine (PtdSer) surface exposure via $\mathrm{Ca}^{2+}$-activated phospholipid scramblase activity $[4,13]$.

Mammalian cell membranes exhibit an asymmetric distribution of phospholipids: sphingomyelin and phosphatidylcholin are enriched at the outer leaflet while phosphatidylserine (PtdSer), phosphatidylethanolamine and phosphatidylinositol reside at the inner leaflet. This asymmetry is maintained by flippases and floppases that mediate selective ATP- dependent translocation of distinct phospholipids. Phospholipid scramblases like TMEM16F and members of the PLSCR (Phospholipid scramblase) or XKR (XK related) protein family aid nondirectional energy-independent transbilayer transport that may ultimately lead to the collapse of membrane asymmetry.

Recently, the lipid scrambling domain of TMEM16F has been characterized [4]. Phospholipid scrambling by TMEM16F can occur during apoptosis or upon cell activation $[7,14,15]$. This effect is essential during platelet activation and bone mineralization by osteoblasts $[1,16-19]$. Here we focused on the effect of TMEM16F on cell viability, proliferation and proapoptotic stimulus response in a Human Embryonic Kidney (HEK) cell model.

\section{Material and Methods}

\section{Cell Culture and imaging}

HEK293 cells (Thermo Scientific, Waltham, MA, USA) were cultivated in standard medium (Dulbecco's modified Eagle medium supplemented with $10 \%$ fetal calf serum and $1 \%$ antibiotics (Pen/Strep) as previously described $[20,21]$. For live cell imaging and photographic growth documentation, cells in culture dishes were examined with an Axio Observer Z1 microscope $\beta$ (Zeiss, Oberkochen, Germany) using Axio Vision 4.7.

Generation of inducible knockdown

The generation of stable cells using lentiviral systems (pInducer21/10) have been described in more detail earlier [20, 21].

Short hairpin RNA was designed with the shRNA prediction algorithm provided at http://biodev. extra.cea.fr/DSIR/DSIR.html [22] which has been described in detail earlier [23]. In total, six different short hairpin RNA fragments were generated and cloned into the pInducer10(RFP) plasmid (Suppl. Fig. 1A; Reverse complement sh1: CTT TCG TCT ACA CTG AGG ACTT; sh2: GGT TAG ATT CGT ATG CTT GTCT; sh3: GTT AGA TTC GTA TGC TTG TCTT; sh4: AGT ACA TCG TGC TTC GTA TTCT; sh5: AAT TTC ACA GAG TAG ATG ACGT; sh6: ATT AAA TTC TTC AAA CTC CGGG). Short hairpin RNA was crosschecked for TMEM16F specificity in silico (Fig. 1D). The resulting pInducer10(RFP) plasmids encoded short hairpin RNA and a red fluorescent protein (RFP) (Fig. 1A-B).

In brief, for generation of stable Doxycycline- inducible knockdown cell lines, HEK293 cells $(10 \mathrm{~cm}$ dish) were simultaneously transfected with $3.5 \mu \mathrm{g}$ pMD2 VSVG (addgene plasmid \#12259), $6.5 \mu \mathrm{g}$ psPAX2 (addgene plasmid \#12260) and $10 \mu \mathrm{g}$ pInducer10shTMEM16F expression plasmids to obtain recombinant virus. The medium of these transiently transfected cells was changed after 6-8 $\mathrm{h}$ and cells were grown for additional $72 \mathrm{~h}$. Then the virus-containing supernatant was collected and filtered through a sterile 0.45 $\mu \mathrm{m}$ syringe driven filter unit (Millipore, Schwalbach am Taunus, Germany). Hence the target HEK293 cells were infected using one volume (up to $2 \mathrm{ml}$ ) of fresh Dulbecco's modified Eagle medium and one volume of the virus-containing filtrate supplemented with polybrene (final concentration $8 \mu \mathrm{g} / \mathrm{ml}$ ). After incubation for $48 \mathrm{~h}$, cells were regenerated in fresh medium for $24 \mathrm{~h}$ and subsequently subjected to selection with Puromycin $4 \mu \mathrm{g} / \mathrm{ml}$.

Expression of short hairpin RNA and RFP was induced by adding $125 \mathrm{ng} / \mathrm{ml}$ Doxycycline to the medium. The knockdown of the target proteinTMEM16F was verified by western blot analysis (Fig.1B). 


\section{Cellular Physiology Cell Physiol Biochem 2016;38:2452-2463 and Biochemistry Published online: June 13,2016 $\quad \begin{aligned} & \text { DOI: 10.1159/000445596 } 2016 \text { The Author(s). Published by S. Karger AG, Basel } \\ & \text { www.karger.com/cpb }\end{aligned}$ Schenk et al:: TMEM16F HEK Viability}

For some experiments, cells were treated with ATP $5 \mathrm{mM}$, Ionomycin $5 \mu \mathrm{M}$ or Staurosporine $1 \mu \mathrm{M}$ (all purchased at Sigma, St. Louis, USA) as indicated.

Preparation of cell lysates and western blot analysis

For western blot analysis, medium was taken off and replaced by $1 \mathrm{x}$ Laemmli (4\% SDS, $5 \%$ 2-mercaptoethanol, $10 \%$ glycerol, $0.002 \%$ bromophenol blue, $0.0625 \mathrm{M}$ Tris-HCl; pH 6.8). After incubation on a shaking plate for $30 \mathrm{~min}$ at room temperature, cell lysates were homogenized via pipetting up and down approximately ten times and then frozen at $-20{ }^{\circ} \mathrm{C}$ for at least $2 \mathrm{~h}$. After boiling for 5 min equal volumes of cell lysates were separated on 8-15\% SDS-PAGE gels (Biorad, Munich, Germany). Proteins were transferred to a PVDF membrane (Millipore) and incubated for $1 \mathrm{~h}$ at room temperature in blocking buffer (5\% BSA powder dissolved in TBS containing $0.05 \%$ Tween-20 (TBS-T)). The lysates were equalized using $\beta$-tubulin (Sigma) or GAPDH (Covance, Princeton, NJ, USA) as loading controls. The TMEM16F antibody was purchased from Sigma (HPA038958), antibodies against Caspase 3 (\#9665), cleaved Capase 3 (Asp175) (\#9664), PARP (\#9542), cleaved PARP (\#5625), ERK1/2 (\#4695), phospho- ERK1/2 (Thr202/Tyr204) (\#43769), AKT (\#9272), phospho- AKT (Thr308) (\#2965), phospho- PDK1 (Ser241) (\#3061), p70S6K (\#2708), phospho p70S6K (Thr389) (\#9234), Bcl2 (\#2876), phospho- (Ser70) Bcl2 (\#2827), GSK3 $\beta$ (\#12456), phospho- GSK3 $\beta$ (Ser9) (\#5558), $\beta$ - Catenin (\#9562) and phospho- $\beta$ - Catenin (Ser33/37/Thr41) (\#9561) were purchased from Cell Signaling (Frankfurt, Germany). The antibody against P2RX7 was a kind gift of Alomone Labs (Jerusalem, Israel). Horseradish peroxidase-conjugated secondary antibodies were purchased from Dianova (Hamburg, Germany). All primary antibodies were used in a 1:1000 dilution in TBS-T and incubated at $4{ }^{\circ} \mathrm{C}$ overnight. After washing three times with TBS-T, the membrane was incubated with horseradish peroxidase-coupled secondary antibodies (Jackson Immunoresearch, Westgrove, PA, USA) diluted 1:5000 (anti mouse) or 1:10000 (anti rabbit) in $5 \%$ BSA powder dissolved in TBS-T for 30$60 \mathrm{~min}$ at room temperature. Afterwards, the blot was washed three times with TBS-T. Western blots were developed with chemiluminescence detection reagent (Roche, Mannheim, Germany). Signals were collected with X-ray film.

Quantification of western blot signals

Developed X-ray films were scanned. Grey scale images were densitometrically quantified using ImageJ (http://rsbweb.nih.gov/ij/).

MTT assay

Cells were grown on translucent flat-bottomed 96- well microtiter plates. On the day of the experiment, cells were provided with $100 \mu \mathrm{l}$ culture medium. For the assay, $10 \mu \mathrm{l}$ tetrazolium MTT (3-(4, 5-dimethylthiazolyl-2)-2, 5-diphenyltetrazolium bromide) were added and cells were incubated at $37{ }^{\circ} \mathrm{C}$ for $30 \mathrm{~min}$. During this time, the yellow tetrazolium salt MTT is converted to purple formazan crystals by intracellular reducing equivalents in metabolically active cells. After $3 \mathrm{~h}$ incubation, $100 \mu \mathrm{l}$ lysis buffer (20 $\%$ sodium dodecyl sulfate, $50 \% \mathrm{~N}, \mathrm{~N}$-dimethylformamide, containing, $0.5 \%$ acetic acid (80\%), $0.4 \% 1 \mathrm{~N}$ HCL) was added to each well and incubated on the shaking plate for $3 \mathrm{~h}$. The optical density was assessed with a fluorescence plate reader (Tecan) at $570 \mathrm{~nm}$.

\section{Flow cytometry}

PtdSer exposure and cell permeabilisation was assessed using flow cytometry. Cells were removed from the culture dishes with ice cold PBS. After centrifugation (4 min ; $1000 \mathrm{xG}$ ), the supernatant was discarded and Annexin V- FITC (BD Biosciences, \#556419) $2.5 \mu \mathrm{l}$ in $50 \mu \mathrm{l}$ staining buffer (containing $1 \%$ bovine serum albumin in $50 \mathrm{mM}$ 2-(4-(2-hydroxyethyl)-1-piperazinyl)-ethansulfonic acid buffer (HEPES), $\mathrm{pH}$ 7.4) was added. After vortexing, the samples were incubated on ice for $20 \mathrm{~min}$. For measurements at the BD Bioscience (FACSCanto I), $450 \mu \mathrm{l}$ staining buffer containing DAPI $2 \mu \mathrm{M}$ were added 5 min prior to the measurement. For measurements at the Becton-Dickinson Fluorescence activated cell sorter (FACSCalibur), propidium iodide (PI) $5 \mu \mathrm{g} / \mathrm{ml}$ was added to the staining buffer. $450 \mu \mathrm{l}$ buffer was added prior to the measurement. A Total of 50000 cells (FACSCanto) or 10000 cells (FACSCalibur) was analyzed. Compensation for the channels (DAPI, FITC, RFP/PE) was calculated using the FACSDiva software (FACScanto) or done manually (FACSCalibur).

\section{KARGER}




\section{Cellular Physiology Cell Physiol Biochem 2016;38:2452-2463 \\ \begin{tabular}{l|l} 
and Biochemistry Published online: June 13, 2016 & $\begin{array}{l}\text { C } 2016 \text { The Author(s). Published by S. Karger AG, Basel } \\
\text { www.karger.com/cpb }\end{array}$
\end{tabular} \\ Schenk et al.: TMEM16F HEK Viability}

Real-time PCR

Real-time PCR was performed using the SYBR Green PCR Master Mix with the ABI PRISM 7900 Sequence Detection System. All instruments and reagents were purchased from Applied Biosystems (Darmstadt, Germany). Relative gene expression values were evaluated with the 2-ddCt method using GAPDH as reference gene. Specific primer pairs:

GAPDH forward AAG ACC TTG GGC TGG GACTG, GAPDH reverse TGG CTC GGC TGG CGAC, TMEM16A forward CCT CAC GGG CTT TGA AGAG TMEM16A reverse CTC CAA GAC TCT GGC TTCGT, TMEM16B forward TGG ATG TGC AAC AAT TGAGA, TMEM16B reverse GCA TTC TGC TGG TCA CACAT, TMEM16F forward CGA ACC CCG GAG TTT GAA GAA, TMEM16F reverse TGC AGG CCA TGA CAG ATA AGG, TMEM16K forward CAG GTC TTC AAA CGT CCAT, TMEM16K reverse TCA TCG TTT CAA AAG CCA ACT.

\section{Statistical analyses}

All data are presented as mean values \pm SEM of at least three independent samples. The number of measurements is given in the legend. Datasets were tested with unpaired t-test or one way ANOVA and Tukey's post test using GraphdPad- Prism 5 (San Diego, CA, USA). Statistical significance was marked * $(\mathrm{P}<$ $0.05),{ }^{* *}(\mathrm{P}<0.01)$ or $* * *(\mathrm{P}<0.001)$.

\section{Results}

Inducible knockdown of TMEM16F led to attenuated growth of HEK293 cells

TMEM16F is endogenously expressed in HEK293 cells as displayed in Fig. 1A. For robust and specific inhibition of TMEM16F function in this cell culture model, an inducible knockdown (KD) was generated employing pInducer10 vector system [24].

Short hairpin RNA shTMEM16F 3 could significantly reduce TMEM16F expression on mRNA and protein level within $48 \mathrm{~h}$ after induction with Doxycycline (Fig. 1B-C). The induction was verified by the expression of the RFP cassette.

In the following, HEK293 shTMEM16F-3 induced with Doxycycline for $5 \mathrm{~d}$ will be referred to as TMEM16F KD.

Asides TMEM16F, HEK293 cells express the Anoctamin family members TMEM16A, TMEM16B and TMEM16K [25]. TMEM16F KD went along with upregulation of TMEM16B and downregulation of TMEM16K (Fig. 1).

Interestingly, after equal seeding, TMEM16F KD cells grew markedly slower than control cells (Fig. 2A-B). Shape and growth pattern appeared unaltered. These observations were confirmed by a significant reduction in a MTT assay after $48 \mathrm{~h}$ induction of knockdown (Fig. 2C).

TMEM16F knockdown increased baseline phosphatidylserine exposure and decreased cell viability

First, we asked the question whether increased apoptosis contributes to the delayed confluency of TMEM16F knockdown cell cultures. Apoptosis goes along with phospholipid scrambling and PtdSer exposition [14] and TMEM16F, among others, functions as a phospholipid scramblase $[4,26]$. Thus Annexin V- FITC staining was employed to test if TMEM16F KD altered the amount of PtdSer exposed on the external leaflet of the plasma membrane. In addition, DAPI was used to detect necrotic cell membrane permeability (schematic overview Fig. 3A-B).

The rate of viable Annexin V and DAPI negative cells was decreased by $9 \%$ in TMEM $16 \mathrm{~F}$ KD cells (Fig. 3C; Q4). Along with this, the Annexin V positive and DAPI negative fraction, widely regarded as apoptotic, was almost doubled (Fig. 3C; Q1). The proportion of DAPI positive necrotic cells averaged 11-13\% and did not differ between the two groups (Fig. 3C; Q2). As depicted in the histogram TMEM16F KD led to a broadening of the Annexin V- FITC affinity rather than increase of a circumscribable Annexin $V$ positive population (Fig. 3D).

To better understand the cell physiological consequence of this increased baseline PtdSer exposure, the sensitivity of TMEM16F KD cells to a proapoptotic stimulus was 


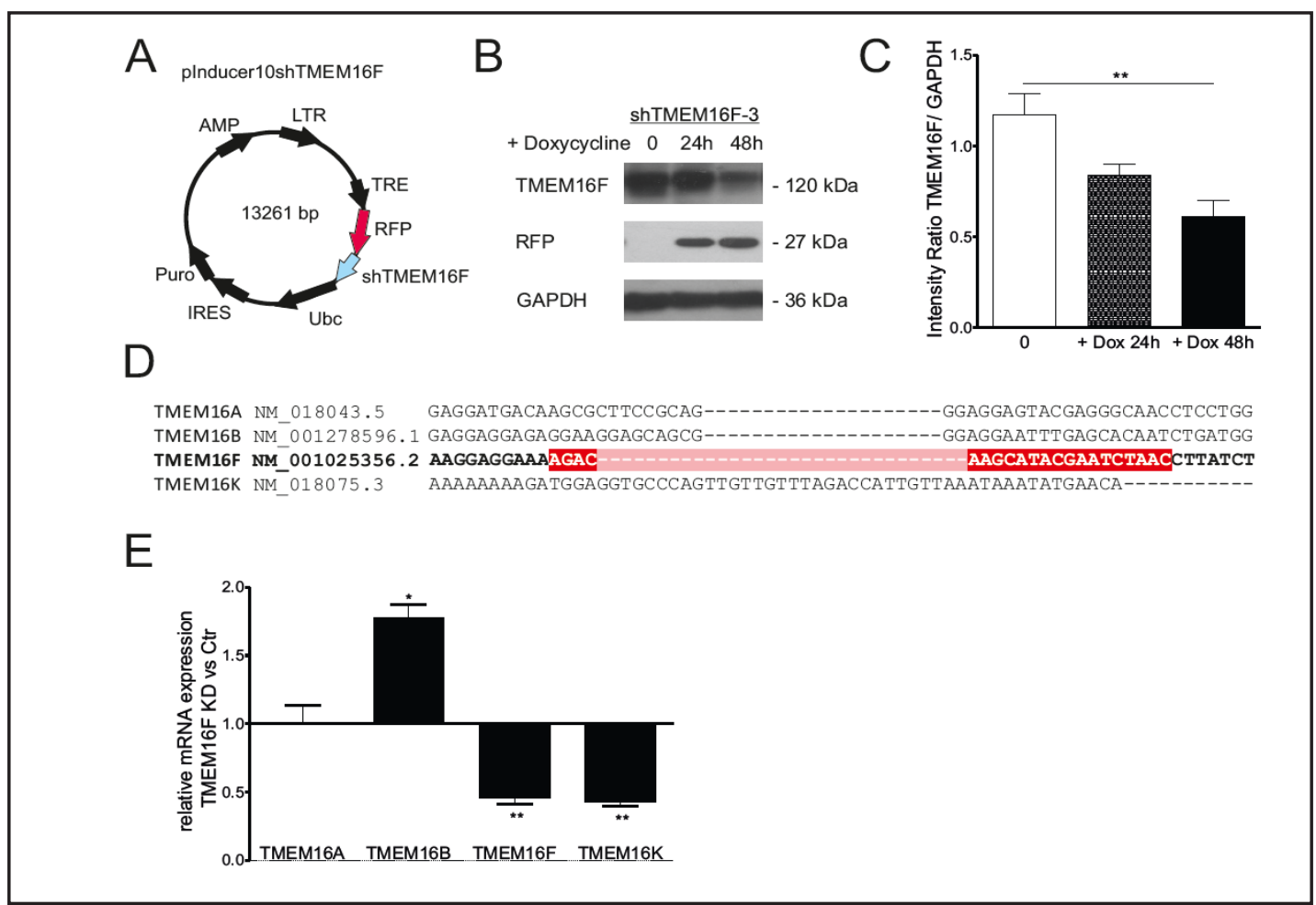

Fig. 1. Generation of an inducible knockdown of TMEM16F in HEK293 cells. (A) A pInducer10shTMEM16F vector system was used. (B) Validation of knockdown efficiency in short hairpin RNA (shTMEM16F-3) expressing HEK293 cells. Upon induction with Doxycycline, RFP and short hairpin RNA were expressed. Lysates were obtained at $0 \mathrm{~h}, 24 \mathrm{~h}$ or $48 \mathrm{~h}$ and probed for GAPDH loading control, induction control RFP and target protein TMEM16F. (C) Quantification of western blot analysis, $n=3$. (D) Alignment of human Anoctamin mRNA sequences at the shTMEM16F-3 target position. (performed with http://www.ebi.ac.uk/ goldman-srv/prank/server/results/prank-S20160109-133734-0636-7638287-oy.html) (E) Quantitative mRNA expression of TMEM16 family members in HEK293 cells. Statistical significance was marked * $(\mathrm{P}<$ $0.05)$, ** $(\mathrm{P}<0.01)$ or $* * *(\mathrm{P}<0.001)$.

assessed. Interestingly, approx. $55 \%$ of both, TMEM16F KD and control cells were viable 3 $\mathrm{h}$ after treatment with the toxic kinase inhibitor Staurosporine (Table 1). The proportion of Annexin V positive TMEM16F KD cells remained stable after this treatment while it almost doubled in control cells.

Taken together, TMEM16F KD led to decreased viability and an increase in baseline PtdSer exposure in HEK293 cells but did not change scrambling in response to kinasedependent apoptotic stimuli.

Different morphological changes, e.g. alterations of size and granularity, precede apoptosis. Asides, a role of TMEM16F in ATP induced PtdSer exposure and ATP induced membrane blebbing has been shown for TMEM16F- and P2RX7 overexpressing HEK293 cells [9]. HEK293 cells endogenously express both proteins. However, FACS analysis did not reveal significant changes in size or granularity after exposure of cells with ATP or ionomycin (Fig. 4).

TMEM16F knockdown resulted in alteration of intracellular signaling pathways

Next we wanted to know how TMEM16F KD affected important intracellular signaling pathways on protein level.

In parallel to the decreased viability seen in flow cytometry TMEM16F KD western blotting detected an increase of cleaved Caspase 3. Cleavage of Poly-ADP-Ribose-Polymerase (PARP) and LC3 I/II, indicator of autophagy, was unaltered (Fig. 5).

\section{KARGER}


Fig. 2. Inducible knockdown of TMEM16F leads to decreased proliferation of HEK293 cells. (A) Growth of HEK293 sh T M E M 16 F - 3 cells. Pictures of TMEM16F knockdown ("+Doxyxcycline") and uninduced control were taken at indicated time points and shown as merged images of light microscopy and RFP. (B) Cell density of HEK293 s h T M E M 16 F - 3 with or without 48 $\mathrm{h}$ induction with Doxycycline after equal seeding. Cell number was coun-

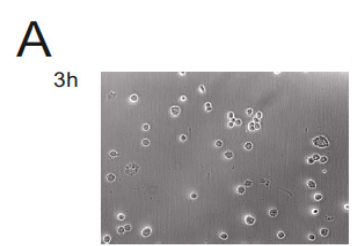

$24 \mathrm{~h}$

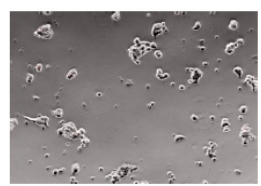

$48 \mathrm{~h}$

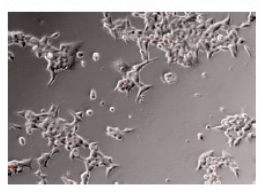

$72 \mathrm{~h}$

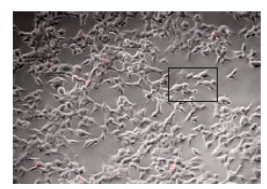

Detailed morphology

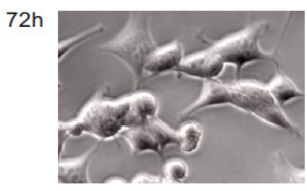

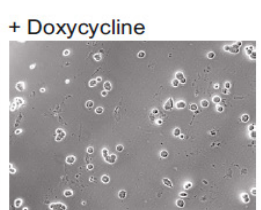
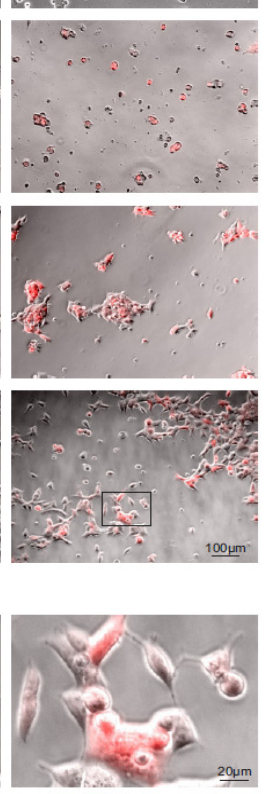

B

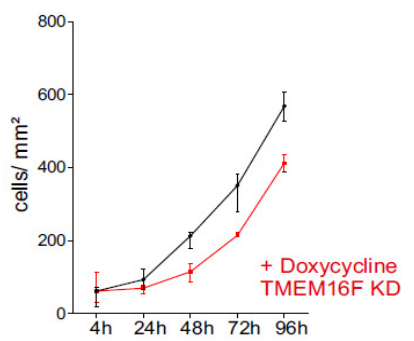

C

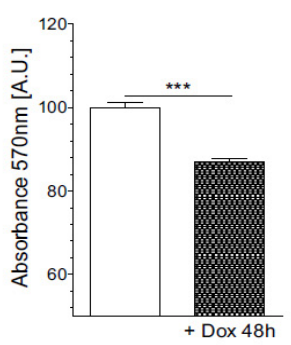
ted in $\mathrm{n}=4$ repre-

sentative pictures. Confluency was reached after $96 \mathrm{~h}$ by uninduced control. (C) MTT assay of HEK293 shTMEM16F-3 with or without $48 \mathrm{~h}$ induction with Doxycycline. Each bar represents a summary of $\mathrm{n}=90$ from 3 individual experiments. Statistical significance was marked ${ }^{*}(\mathrm{P}<0.05),{ }^{* *}(\mathrm{P}<0.01)$ or ${ }^{* * *}(\mathrm{P}<0.001)$.

Next, we examined kinase signaling and found that phosphorylation of ERK was unchanged. In contrast, phosphorylation of AKT at the activating T308-site was increased (Fig. 6A) while the total abundance and phosphorylation of AKT at S473 were unaltered. Activation of AKT at phospho- T308 can be conferred by PDK1, and in accordance to our observations phospho- PDK1 was increased in TMEM16F KD. To address AKT signaling in more detail, we evaluated different downstream targets. The increased phosphorylation of AKT was accompanied by decreased phospho- S9 GSK3 $\beta$ level and decreased phospho$\beta$ - Catenin. Phosphorylation of $\mathrm{Bcl} 2$, a potential pro-survival signal, was only marginally increased while an accumulation of Cyclin D1 indicative of pro-proliferative signaling was visible. Phosphorylation of p70S6K representative of potential activation of mTOR signaling remained unaltered (Fig. 6B).

Since knockdown of TMEM16F was achieved by a doxycycline- inducible vector system we verified that doxycycline treatment alone did not regulate these signaling pathways in HEK293 cells (Fig. 6C).

Figure 7 gives an overview of the intracellular signaling pathways that have been found regulated in TMEM16F KD.

\section{Discussion}

TMEM16F is expressed in a variety of tissues and holds important functions in blood coagulation, for immune cells, bone homeostasis and epithelial organs. Not only the wide 
Fig. 3. Flow cytometry shows increased phosphatidylserine exposure and decreased cell viability of TMEM16F knockdown cells. (A) Annexin V- FITC and DAPI staining were used for detection of phosphatidylserine exposure or necrotic membrane permeabilisation respectively. (B) Representative dot plot of Annexin V- FITC and DAPI staining in HEK pInducer10shTMEM16F3 cells including the gates used for following analyses. Left graphic: Control cells, right graphic: TMEM16F knockdown (KD). (C) Percent of cells gated in the indicated quadrant in $\mathrm{KD}$ and uninduced control $(n=3$, respresented as mean \pm SEM). Statistical significance was marked $*$ (P<0.05), ** $(\mathrm{P}<0.01)$ or $* * *(\mathrm{P}<0.001)$. (D) Normalized histograms for Annexin V- FITC positive cells in $\mathrm{KD}$ and uninduced control.

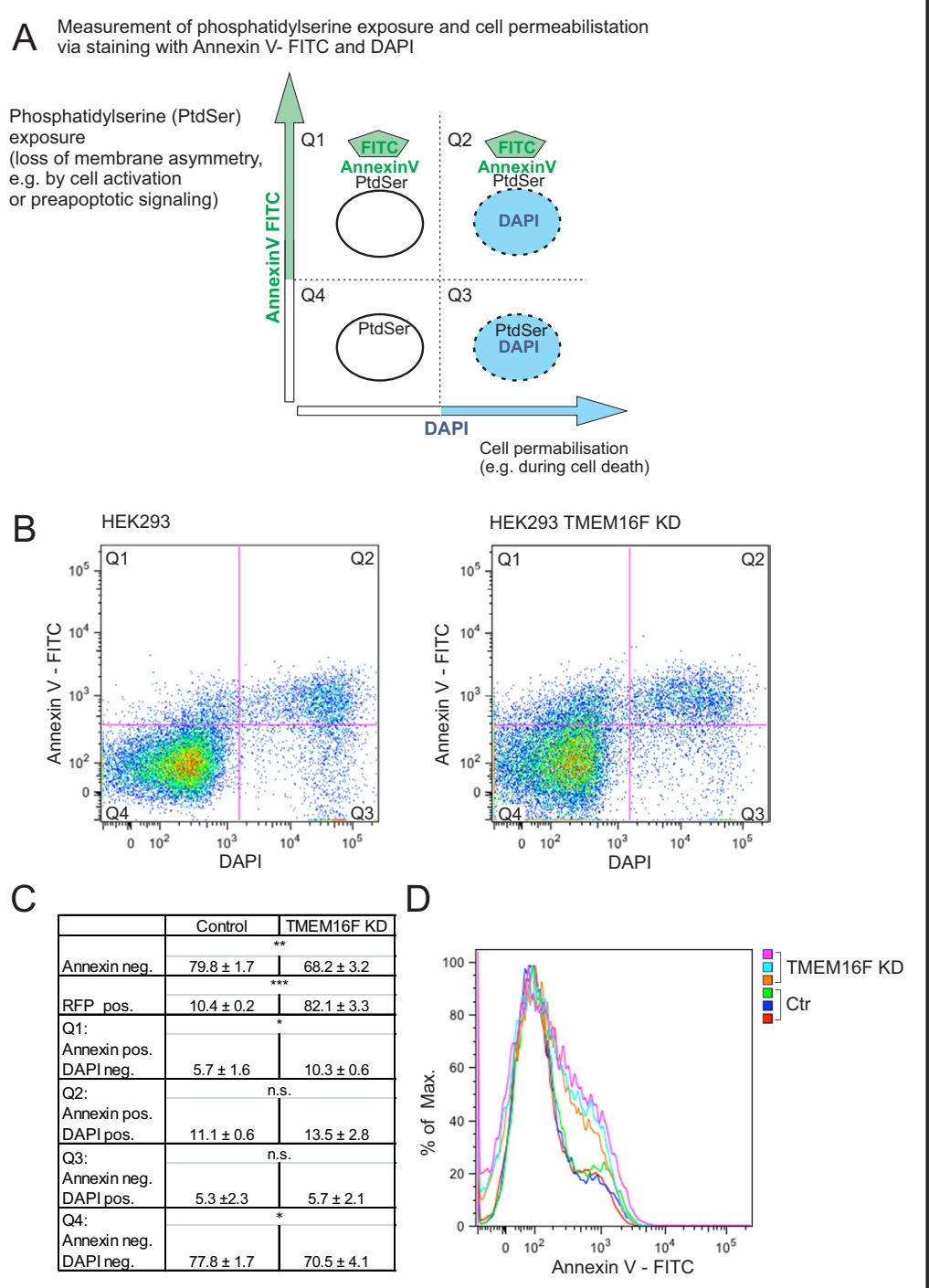

Table 1. Flow cytometric detection of phosphatidylserine exposure in TMEM16F knockdown cells after treatment with Staurosporine. Annexin V FITC and DAPI staining were used for detection of phosphatidylserine exposure or necrotic membrane permeabilisation respectively. The table lists the percentage of TMEM16F knockdown (KD) cells (HEK293 pInducer shTMEM16F + Doxycycline (125 ng/ml, $110 \mathrm{~h})$ ) and uninduced control cells gated in the indicated quadrant $(n=3$, respresented as mean \pm SEM). Statistical significance between TMEM16F KD and control cells (t-test) was marked * $(\mathrm{P}<0,05),{ }^{* *}(\mathrm{P}<0,01)$ or *** $(\mathrm{P}<0,001)$

\begin{tabular}{|c|c|c|c|c|}
\hline & \multicolumn{2}{|c|}{ DMSO } & \multicolumn{2}{|c|}{ Staurosporine $1 \mu \mathrm{M}(3 \mathrm{~h})$} \\
\hline & Control & TMEM16F KD & Control & TMEM16F KD \\
\hline \multirow[t]{2}{*}{ Q1: Annexin pos. DAPI neg. } & \multicolumn{2}{|r|}{ ** } & \multicolumn{2}{|r|}{ * } \\
\hline & $3,7 \pm 0,5$ & $12,1 \pm 1,8$ & $6,9 \pm 0,5$ & $10,5 \pm 1,8$ \\
\hline \multicolumn{5}{|l|}{ Q2: Annexin pos. DAPI pos. } \\
\hline & $11,0 \pm 0,6$ & $13,8 \pm 2,1$ & $18,6 \pm 1,0$ & $19,3 \pm 4,4$ \\
\hline \multicolumn{5}{|l|}{ Q3: Annexin neg. DAPI pos. } \\
\hline & $12,0 \pm 1,7$ & $9,7 \pm 0,5$ & $18,6 \pm 1,1$ & $16,5 \pm 2,4$ \\
\hline \multirow[t]{2}{*}{ Q4: Annexin neg. DAPI neg. } & & * & & \\
\hline & $73,3 \pm 1,6$ & $64,5 \pm 4,2$ & $55,9 \pm 2,3$ & $53,7 \pm 6,7$ \\
\hline
\end{tabular}




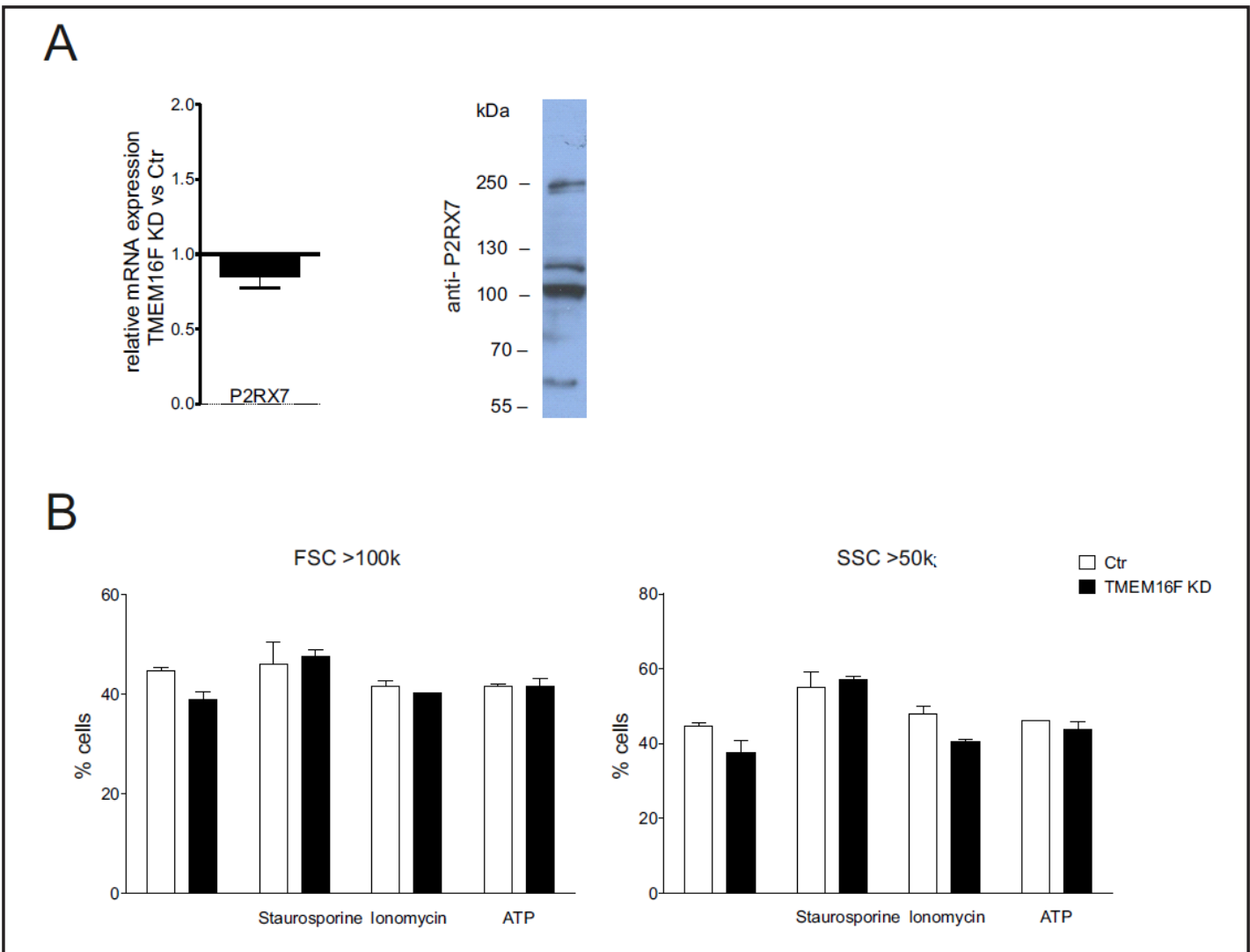

Fig. 4. Size and granularity are unaltered in TMEM16F knockdown cells. (A) P2RX7 expression in HEK293 cells on mRNA and protein level as verified with quantitative PCR and western blot analyses. (B) FACS measurement of size and granularity. FSC correlates with the size of cells, SSC correlates with granularity of cells. To facilitate the calculations, cutoff values approximating the shoulder of the histogram were introduced (FSC $>100 \mathrm{k}$, SSC $>50 \mathrm{k}$ ).

distribution in the human body but also its diverse, incompletely deciphered functions bring out TMEM16F as an interesting player in intracellular signaling. The present study chose human kidney cells as a model and focused on TMEM16F influence on cell viability.

In parallel to a study in myoblasts [27] we could show that knockdown of TMEM16F decreased the growth of HEK293 cells. Our results suggest that this attenuation may be attributable to decreased cell viability.

So far, it has been proposed that phospholipid scrambling by TMEM16F is not primarily involved in apoptosis but accounts for PtdSer exposure upon cell activation. One might expect that under resting conditions, PtdSer exposure by phospholipid scrambling was at a low level and that this would rather be lowered by TMEM16F KD. In contrast, we found increased baseline PtdSer exposure in TMEM16F KD. With attention to the decreased viability, increased PtdSer exposure may be culprit or consequence. However, if PtdSer exposure was the result of a proapoptotic signaling cascade one might expect a "Make-or-Break" signal resulting in strong instantaneous PtdSer exposure and a circumscribable Annexin V- positive population in flow cytometry. But TMEM16F KD led to gradual loss of the asymmetric distribution of PtdSer between the membrane leaflets with PtdSer residing at the inner leaflet.

Interestingly, TMEM16F KD did not sensitize the cells to Staurosporine- induced cell death. This correlates with other groups' observation of a marked decrease in death- and activation-induced phospholipid scrambling $[16,28,29]$. Recently beneficial effects of anion channel blockade have been shown in a rat model of ischemia reperfusion [30]. 


\section{Cellular Physiology Cell Physiol Biochem 2016;38:2452-2463 \begin{tabular}{ll|l} 
DOI: 10.1159/000445596 & O 2016 The Author(s). Published by S. Karger AG, Basel \\
www.karger.com/cpb
\end{tabular} Schenk et al.: TMEM16F HEK Viability}

Fig. 5. TMEM16F knockdown leads to induction of apoptosis. Western blot analyses of three independent lysates from HEK293 shTMEM16F3 cells. Samples that have been induced with Doxycycline for $96 \mathrm{~h}$ are indicated as TMEM16F KD. Equal loading, efficient induction and knockdown of the target protein TMEM16F were verified and western blots of indicator proteins of apoptosis (cleavage of Caspase 3 and PARP) and the autophagic pathway (LC3 I/II) were performed. Statistical significance was marked * $(\mathrm{P}<0.05)$, ** $(\mathrm{P}<0.01)$ or $* * *(\mathrm{P}<0.001)$.

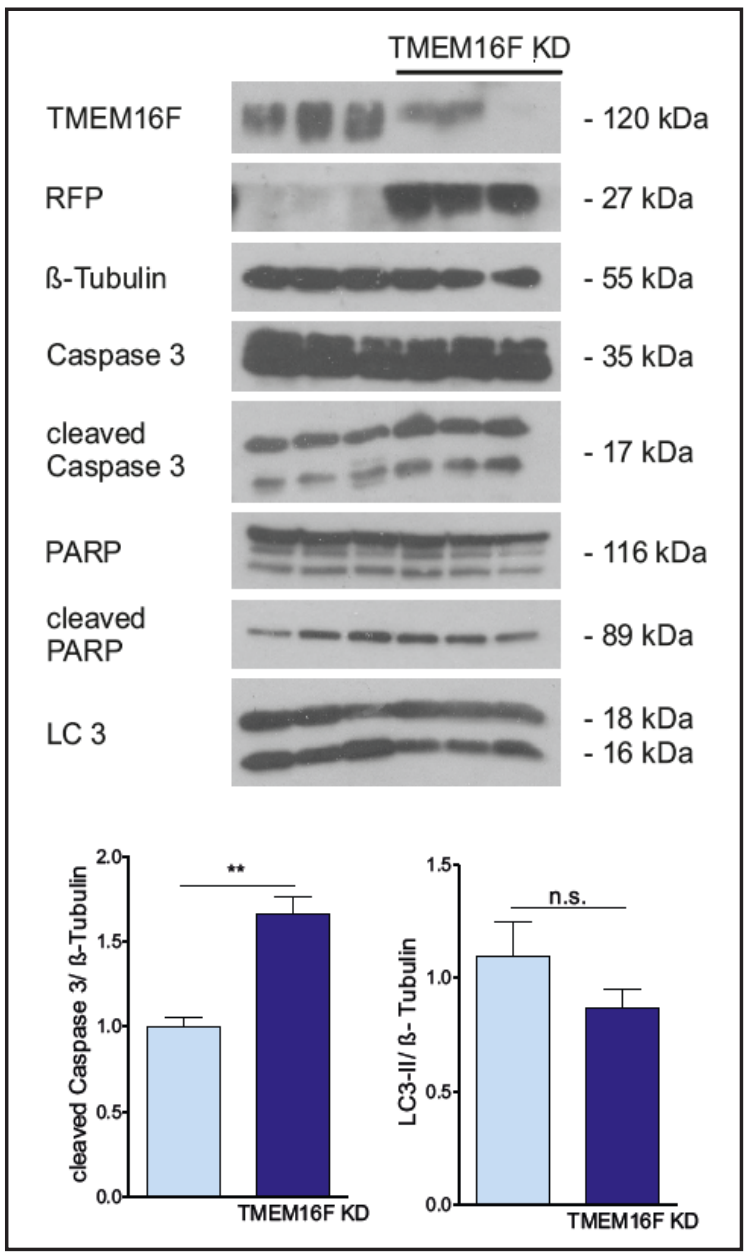

This may be understood on background of the diverse functions: TMEM16F is known to promote membrane lipid redistribution and known to be involved in preapoptotic processes such as volume decrease and membrane blebbing [6, 9, 31].

Intracellular proapoptotic signaling such as Caspase activation was paralleled by activation of prosurvival and pro- proliferative signaling molecules such as $\beta$ - Catenin. This might contribute to the unchanged susceptibility to Staurosporine- induced cell death.

The pro-survival signaling in TMEM16F KD might be triggered by AKT signaling. These pathways might differ between different cell types: While in HEK293 cells decreased proliferation correlated not only with activation of Caspase 3 but also with increased phosphorylation of AKT at T308 and activation of $\beta$ - Catenin signaling and thereby may disrupt the cell cycle [32], in C2C12 myoblasts, reduced proliferation was paralleled by decreased phosphorylation of AKT and ERK [27].

Taken together our study indicates that TMEM16F regulated viability and proliferation of Human Kidney cells via activation of AKT and might modulate the function of phospholipid sorting molecules in the plasma membrane. Further studies are necessary to elucidate the ambiguous role of TMEM16F in apoptosis, viability and membrane phospholipid sorting.

\section{Acknowledgements}

This work was supported by Else Kröner-Fresenius Stiftung. The authors thank Sandra Kohaus and Eva Mickholz for technical support. We acknowledge support by Open Access Publication Fund of University of Muenster. 


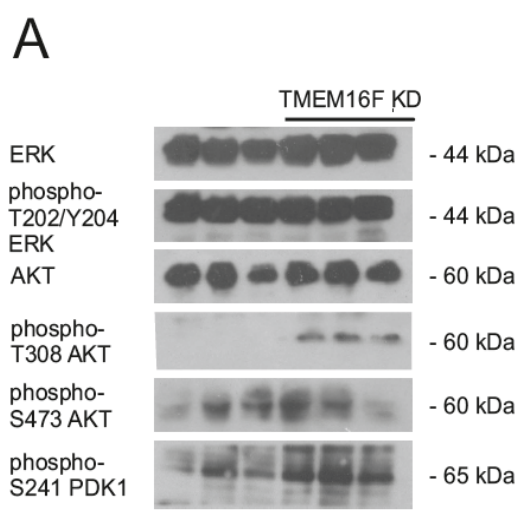

B

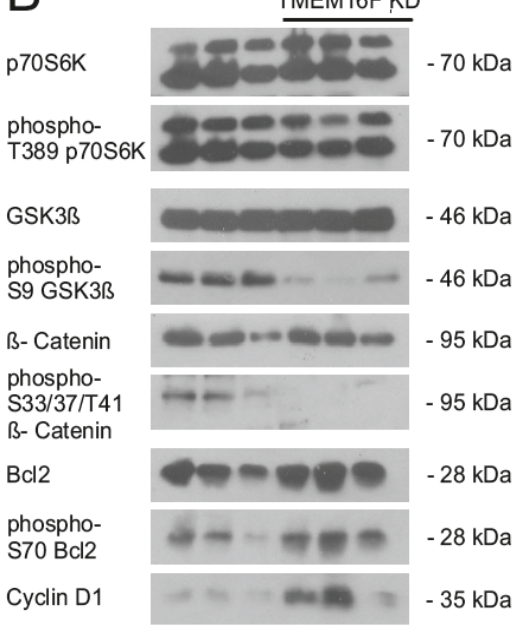

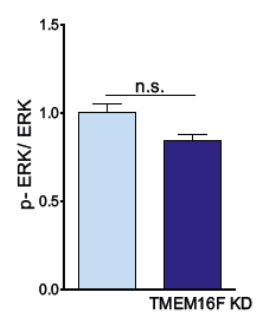
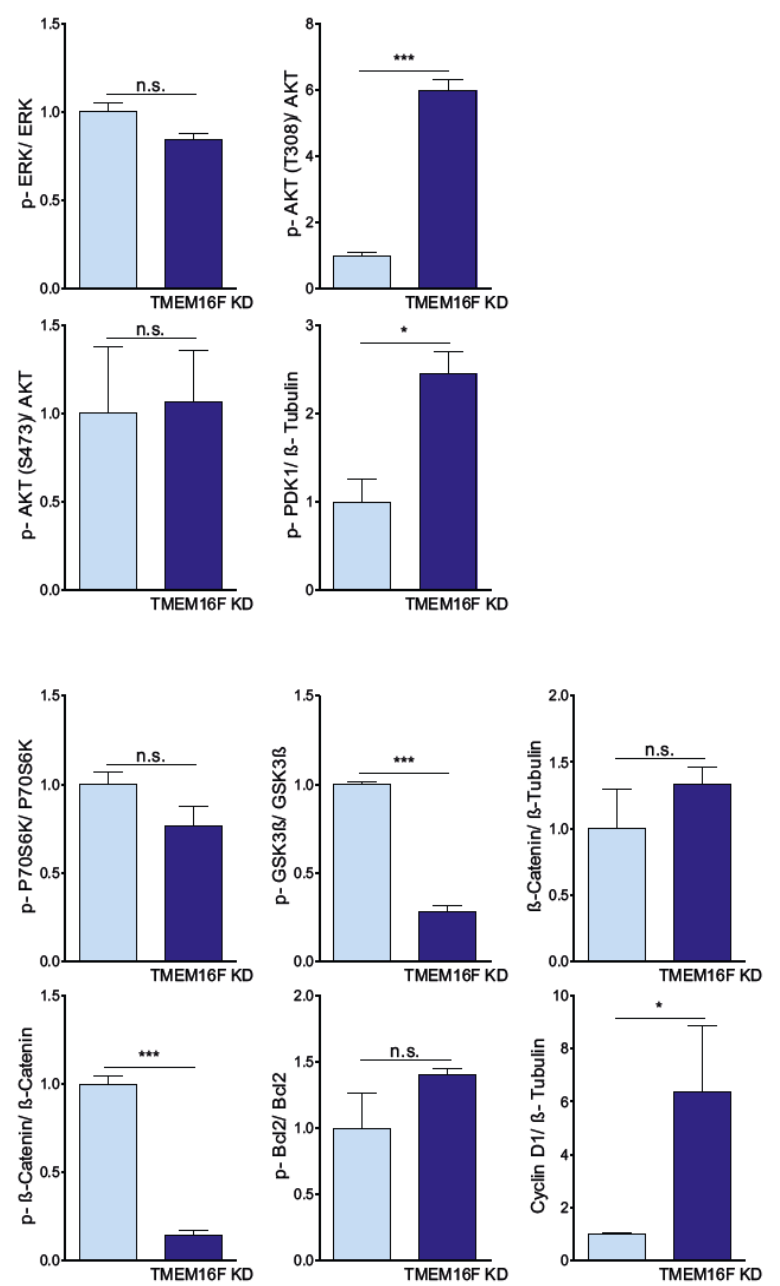

\section{HEK plnd10shTMEM16F-3 HEK wildtype TMEM16F KD $[+\operatorname{Dox}(7 d)]$}

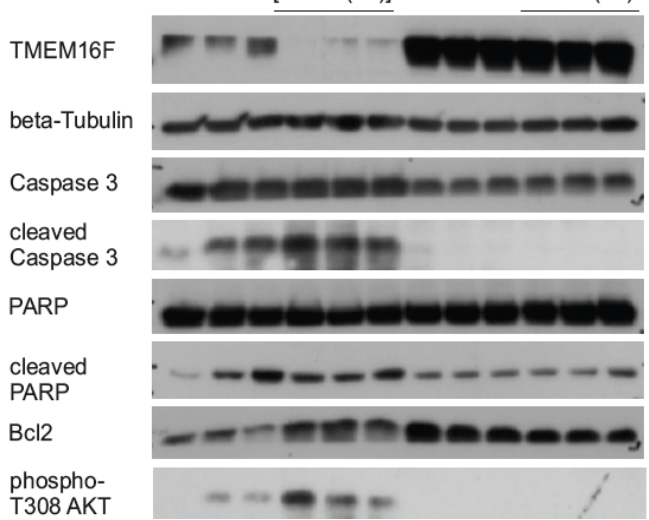

Fig. 6. TMEM16F knockdown leads activation of AKT signaling. Western blot analyses of three independent lysates from HEK293 shTMEM16F3 cells. Samples that have been induced with Doxycycline for $96 \mathrm{~h}$ are indicated as TMEM16F KD. (A) Western blots of ERK, AKT and their phosphorylated forms. (B) Further, representatives of AKT- and mTOR- signaling pathway were scrutinized. (C) Western blot analyses of three independent lysates from TMEM16F KD and Doxycycline treated wildtype HEK293 cells. Statistical significance was marked $^{*}(\mathrm{P}<0.05)$, ** $(\mathrm{P}<0.01)$ or *** $(\mathrm{P}<0.001)$. 


\section{Cellular Physiology Cell Physiol Biochem 2016;38:2452-2463 \begin{tabular}{l|l} 
DOI: 10.1159/000445596 & O 2016 The Author(s). Published by S. Karger AG, Basel \\
www.karger.com/cpb
\end{tabular}

Fig. 7. Schematic representation of HEK293 cell intracellular signaling after TMEM16F knockdown. The green line represents phosphatidylserine cell surface exposition. Several signaling pathways are regulated suggesting differentiated role for TMEM16F in survival and apoptosis.

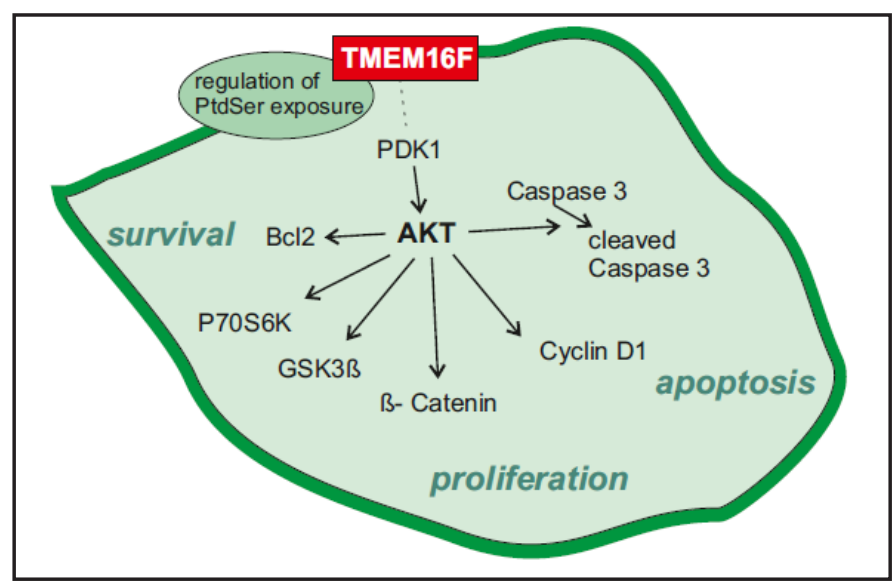

\section{Disclosure Statement}

The authors declare no conflict of interest.

\section{References}

1 Fujii T, Sakata A, Nishimura S, Eto K, Nagata S: TMEM16F is required for phosphatidylserine exposure and microparticle release in activated mouse platelets. Proc Natl Acad Sci USA 2015;112:12800-12805.

2 Yang H, Kim A, David T, Palmer D, Jin T, Tien J, Huang F, Cheng T, Coughlin SR, Jan YN, Jan LY: TMEM16F forms a Ca2+-activated cation channel required for lipid scrambling in platelets during blood coagulation. Cell 2012;151:111-122.

3 Martins JR, Faria D, Kongsuphol P, Reisch B, Schreiber R, Kunzelmann K: Anoctamin 6 is an essential component of the outwardly rectifying chloride channel. Proc Natl Acad Sci USA 2011;108:18168-18172.

4 Yu K, Whitlock JM, Lee K, Ortlund EA, Yuan Cui Y, Hartzell HC: Identification of a lipid scrambling domain in AN06/TMEM16F. Elife 2015;4:10.7554/eLife.06901.

5 Grubb S, Poulsen KA, Juul CA, Kyed T, Klausen TK, Larsen EH, Hoffmann EK: TMEM16F (Anoctamin 6), an anion channel of delayed $\mathrm{Ca}(2+)$ activation. J Gen Physiol 2013;141:585-600.

6 Juul CA, Grubb S, Poulsen KA, Kyed T, Hashem N, Lambert IH, Larsen EH, Hoffmann EK: Anoctamin 6 differs from VRAC and VSOAC but is involved in apoptosis and supports volume regulation in the presence of Ca2+. Pflugers Arch 2014;466:1899-1910.

7 Kmit A, van Kruchten R, Ousingsawat J, Mattheij NJ, Senden-Gijsbers B, Heemskerk JW, Schreiber R, Bevers EM, Kunzelmann K: Calcium-activated and apoptotic phospholipid scrambling induced by Ano6 can occur independently of Ano6 ion currents. Cell Death Dis 2013;4:e611.

8 Ousingsawat J, Wanitchakool P, Schreiber R, Wuelling M, Vortkamp A, Kunzelmann K: Anoctamin-6 controls bone mineralization by activating the calcium transporter NCX1. J Biol Chem 2015;290:6270-6280.

9 Ousingsawat J, Wanitchakool P, Kmit A, Romao AM, Jantarajit W, Schreiber R, Kunzelmann K: Anoctamin 6 mediates effects essential for innate immunity downstream of P2X7 receptors in macrophages. Nat Commun 2015;6:6245.

10 Schreiber R, Faria D, Skryabin BV, Wanitchakool P, Rock JR, Kunzelmann K: Anoctamins support calciumdependent chloride secretion by facilitating calcium signaling in adult mouse intestine. Pflugers Arch 2015;467:1203-1213.

11 Kunzelmann K, Cabrita I, Wanitchakool P, Ousingsawat J, Sirianant L, Benedetto R, Schreiber R: Modulating Ca signals: a common theme for TMEM16, Ist2, and TMC. Pflugers Arch 2016;468:475-490.

12 Scudieri P, Caci E, Venturini A, Sondo E, Pianigiani G, Marchetti C, Ravazzolo R, Pagani F, Galietta LJ: Ion channel and lipid scramblase activity associated with expression of TMEM16F/ANO6 isoforms. J Physiol 2015;593:3829-3848.

13 Segawa K, Suzuki J, Nagata S: Constitutive exposure of phosphatidylserine on viable cells. Proc Natl Acad Sci USA 2011;108:19246-19251. 


\section{Cellular Physiology Cell Physiol Biochem 2016;38:2452-2463 \begin{tabular}{l|l} 
DOI: 10.1159/000445596 & $\begin{array}{l}\text { O 2016 The Author(s). Published by S. Karger AG, Basel } \\
\text { www.karger.com/cpb }\end{array}$
\end{tabular} \\ Schenk et al.: TMEM16F HEK Viability}

14 Kodigepalli KM, Bowers K, Sharp A, Nanjundan M: Roles and regulation of phospholipid scramblases. FEBS Lett 2015;589:3-14.

15 Kunzelmann K: Ion channels in regulated cell death. Cell Mol Life Sci DOI:10.1007/s00018-016-2208-z.

16 Liu G, Liu G, Chen H, Borst O, Gawaz M, Vortkamp A, Schreiber R, Kunzelmann K, Lang F: Involvement of Ca2+ Activated Cl- Channel Ano6 in Platelet Activation and Apoptosis. Cell Physiol Biochem 2015;37:19341944.

17 van Kruchten R, Mattheij NJ, Saunders C, Feijge MA, Swieringa F, Wolfs JL, Collins PW, Heemskerk JW, Bevers EM: Both TMEM16F-dependent and TMEM16F-independent pathways contribute to phosphatidylserine exposure in platelet apoptosis and platelet activation. Blood 2013;121:1850-1857.

18 Harper MT, Poole AW: Chloride channels are necessary for full platelet phosphatidylserine exposure and procoagulant activity. Cell Death Dis 2013;4:e969.

19 Ehlen HW, Chinenkova M, Moser M, Munter HM, Krause Y, Gross S, Brachvogel B, Wuelling M, Kornak U, Vortkamp A: Inactivation of anoctamin-6/Tmem16f, a regulator of phosphatidylserine scrambling in osteoblasts, leads to decreased mineral deposition in skeletal tissues. J Bone Miner Res 2013;28:246-259.

20 Schulze U, Vollenbroker B, Braun DA, Van Le T, Granado D, Kremerskothen J, Franzel B, Klosowski R, Barth J, Fufezan C, Wolters DA, Pavenstadt H, Weide T: The Vac14-interaction network is linked to regulators of the endolysosomal and autophagic pathway. Mol Cell Proteomics 2014;13:1397-1411.

21 Wennmann DO, Vollenbroker B, Eckart AK, Bonse J, Erdmann F, Wolters DA, Schenk LK, Schulze U, Kremerskothen J, Weide T, Pavenstadt H: The Hippo pathway is controlled by Angiotensin II signaling and its reactivation induces apoptosis in podocytes. Cell Death Dis 2014;5:e1519.

22 Vert JP, Foveau N, Lajaunie C, Vandenbrouck Y: An accurate and interpretable model for siRNA efficacy prediction. BMC Bioinformatics 2006;7:520.

23 Dow LE, Premsrirut PK, Zuber J, Fellmann C, McJunkin K, Miething C, Park Y, Dickins RA, Hannon GJ, Lowe SW: A pipeline for the generation of shRNA transgenic mice. Nat Protoc 2012;7:374-393.

24 Meerbrey KL, Hu G, Kessler JD, Roarty K, Li MZ, Fang JE, Herschkowitz JI, Burrows AE, Ciccia A, Sun T, Schmitt EM, Bernardi RJ, Fu X, Bland CS, Cooper TA, Schiff R, Rosen JM, Westbrook TF, Elledge SJ: The pINDUCER lentiviral toolkit for inducible RNA interference in vitro and in vivo. Proc Natl Acad Sci USA 2011;108:3665-3670.

25 Thakur SS, Geiger T, Chatterjee B, Bandilla P, Frohlich F, Cox J, Mann M: Deep and highly sensitive proteome coverage by LC-MS/MS without prefractionation. Mol Cell Proteomics 2011;10:M110.003699.

26 Lhermusier T, Chap H, Payrastre B: Platelet membrane phospholipid asymmetry: from the characterization of a scramblase activity to the identification of an essential protein mutated in Scott syndrome. J Thromb Haemost 2011;9:1883-1891.

27 Zhao P, Torcaso A, Mariano A, Xu L, Mohsin S, Zhao L, Han R: Anoctamin 6 regulates C2C12 myoblast proliferation. PLoS One 2014;9:e92749.

28 Brooks MB, Catalfamo JL, MacNguyen R, Tim D, Fancher S, McCardle JA: A TMEM16F Point Mutation Causes an Absence of Canine Platelet TMEM16F and Ineffective Activation and Death-Induced Phospholipid Scrambling. J Thromb Haemost 2015;13:2240-2252.

29 Forschbach V, Goppelt-Struebe M, Kunzelmann K, Schreiber R, Piedagnel R, Kraus A, Eckardt KU, Buchholz B: Anoctamin 6 is localized in the primary cilium of renal tubular cells and is involved in apoptosisdependent cyst lumen formation. Cell Death Dis 2015;6:e1899.

30 Wang X, Cao Y, Shen M, Wang B, Zhang W, Liu Y, He X, Wang L, Xia Y, Ding M, Xu X, Ren J: DIDS reduces ischemia/reperfusion-induced myocardial injury in rats. Cell Physiol Biochem 2015;35:676-688.

31 Sirianant L, Ousingsawat J, Wanitchakool P, Schreiber R, Kunzelmann K: Cellular volume regulation by anoctamin 6: Ca, phospholipase A2 and osmosensing. Pflugers Arch 2016;468:335-349.

32 Shimura T, Kobayashi J, Komatsu K, Kunugita N: DNA damage signaling guards against perturbation of cyclin D1 expression triggered by low-dose long-term fractionated radiation. Oncogenesis 2014;3:e132. 